\title{
EL SISTEMADEINVERSIÓN PÚBLICA (INVIERTE.PE) Y LOS DESAFÍOS QUE ENFRENTA PARA MEJORAR LAEJECUCIÓN DE LA INVERSIÓN PÚBLICA: ¿UN ERROR DE DIAGNÓSTICO?
}

\author{
Alfonso Jesús Garcés Manyari
}

\section{Resumen}

La inversión pública en el Perú, en el último quinquenio, ha venido reportando los niveles más bajos de ejecución de inversión de, por lo menos, los últimos quince años, a pesar de haberse modificado el sistema de inversión pública (Invierte.pe, que reemplazó al SNIP), luego del anuncio del nuevo esquema. Entonces, ¿qué pasó? ¿Por qué, si se eliminó el SNIP por el Invierte.pe, no se tradujo ese cambio en una mejora de la inversión pública? ¿Fue un error de diagnóstico? En el presente trabajo, buscamos encontrar una respuesta que trate de explicar los bajos niveles de ejecución de la inversión pública que se viene reportando en los tres niveles de gobierno, así como algunas propuestas de mejora.

\section{INTRODUCCIÓN}

La pandemia de la COVID-19 ha traído consigo, además de una crisis sanitaria sin precedentes en el Perú, una crisis económica y financiera, similares a las que nos dejó la guerra con Chile. Ha arrasado con todo lo que se ha cruzado por su paso, lo que implica que el Estado deba implementar una política contracíclica, haciendo más expansivo el gasto público, a través de un shock de inversiones. Sin embargo, si uno revisa los magros resultados de la ejecución presupuestal vistos luego de la "reforma" del sistema de inversión pública implementada a partir del 2017, se aprecia una caída sostenida de esta que haría poco viable ese shock de inversiones.

Profesor de la Maestría de Gestión Pública de la Universidad San Ignacio de Loyola y de la Maestría en Derecho Administrativo Económico de la Universidad Continental. Socio de Guzmán Napurí \& Segura Abogados. Ha sido jefe de Gabinete de Asesores del MEF y viceministro de Justicia. Es Máster en Economía, Regulación y competencia en los Servicios Públicos por la Universidad de Barcelona y un Postgrado en Compliance por la Universidad Pompeu Fabra y por la Universidad Carlos III de Madrid 
Así, si uno revisa las cifras oficiales, a través de la consulta amigable del Ministerio de Economía y Finanzas (MEF) (Gráfico 1)., puede advertir que la ejecución promedio de la inversión pública, por todo nivel de gobierno, en el período 2017-2020 asciende a 64,7 \%; mientras que en el período 2012-2015, la ejecución promedio fue de 74,3\%, es decir, luego de aprobarse el nuevo sistema de inversión pública, el Perú cayó casi 10pp.

\section{Gráfico 1}

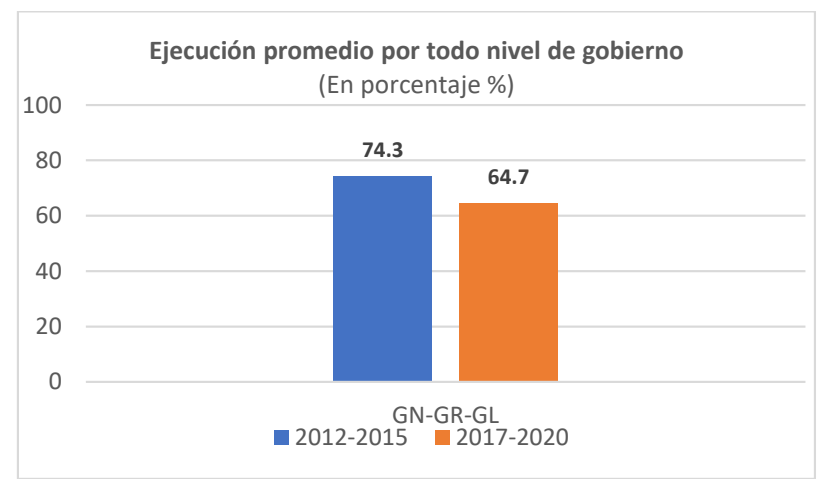

Fuente: Consulta amigable MEF Elaboración propia

Si analizamos la ejecución por nivel de gobierno, encontramos que en el período 20122015, el gobierno nacional tuvo una ejecución promedio de $82,5 \%$, mientras que en el período 2017-2020 apenas llegó al 75,6 \%, es decir, cayó la ejecución en $5 \%$. En el caso de los gobiernos regionales, la situación es más dramática. Por ejemplo, en el período 20122015 , estos alcanzaron un promedio de 76,7 $\%$, mientras que en el período 2017-2020, solo ejecutaron $60,4 \%$, es decir, retrocedió en más de $15 \mathrm{pp}$. En el caso de los gobiernos locales, se registró una caída superior al $10 \%$. Pasando de un 69,3\% en el 2012-2015 a un $58,3 \%$ en el período 2017-2020, tal como se observa en el Gráfico 2.
Gráfico 2

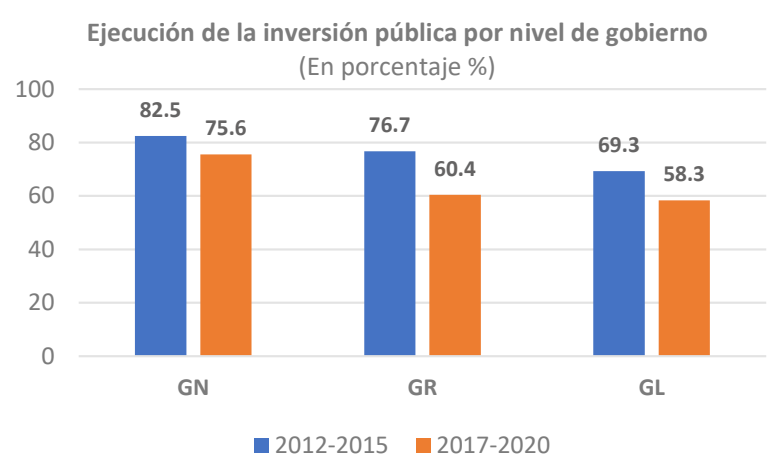

Fuente: Consulta amigable MEF

Elaboración propia

Si observamos el nivel de ejecución promedio de la inversión pública, por todo nivel de gobierno, de los últimos quince años, apreciamos que uno de los picos más bajo ha sido durante la vigencia del invierte.pe (año 2017 hasta la actualidad), siendo el pico de mayor ejecución el año 2015. Asimismo, la tendencia de ejecución promedio de la inversión pública del periodo analizado tiene un comportamiento polinómico de grado 6, conforme el Gráfico 3.

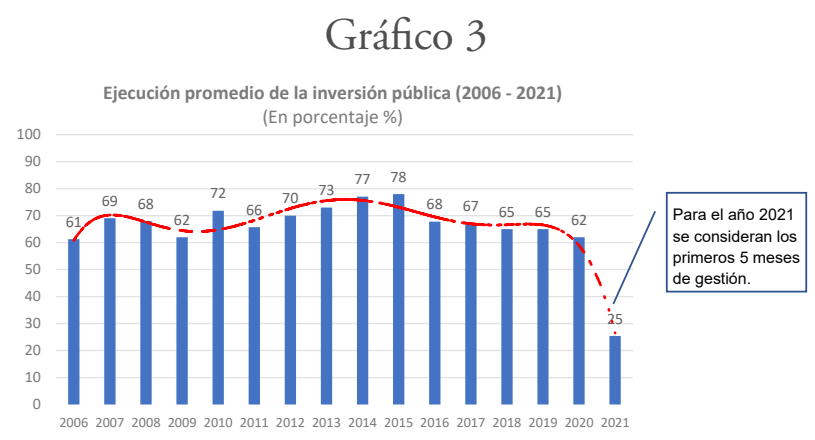

Fuente: Consulta amigable MEF

Elaboración propia

Si comparamos el nivel de ejecución de la inversión pública de los primeros cinco meses de los años electorales, tendremos que en el 2021, dada la alta necesidad de infraestructura y de proyectos de inversión necesarios para enfrentar la pandemia originada por la COVID-19, 
tuvo el mejor desempeño en el mismo período, tal como se advierte en el Gráfico 4.

\section{Gráfico 4}

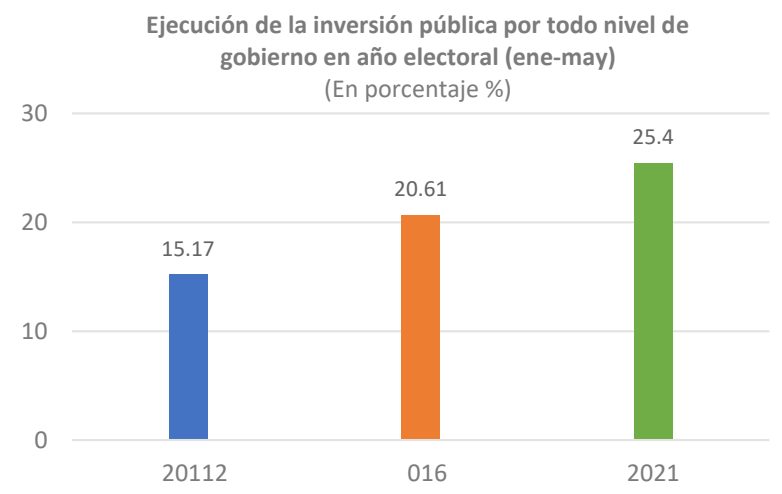

Fuente: Consulta amigable MEF

Elaboración propia

Entonces ¿cómo se puede aplicar una política contracíclica efectiva y orientada a generar un fuerte shock de empleo, a través de un mayor gasto en inversión pública a nivel nacional, si a pesar del reemplazo del SNIP con el invierte. pe partir del año 2017, ha venido cayendo de manera sostenida los niveles de ejecución de inversión? Intentaremos responder dicha interrogante a lo largo del presente artículo. Veremos si se trata de un problema de diagnóstico que llevó al país a un desplome sostenido de la ejecución de la inversión pública en los últimos cuatro años, comparativamente con un crecimiento sostenido de la ejecución de la inversión en el periodo 2012 al 2015.

\section{LA FALACIA DEL SISTEMA DE INVERSIÓN PÚBLICA COMO PRINCIPAL PROBLEMA DE LASUBEJECUCIÓNDE INVERSIONES}

Como ya hemos señalado, la inversión pública pasa por su peor momento, contribuida en parte por una evaluación parcial de la problemática, que se vio agudizada con la pandemia provocada por la COVID-19, y que conspiró para tener como resultado una reforma del sistema de inversión pública que a, la luz de los resultados, no ha cumplido con los objetivos trazados inicialmente. En ese sentido, podemos afirmar, sin a partir de los datos analizados, que la inversión pública en el Perú se encuentra en cuidados intensivos.

Por ello, se requiere dar una mirada integral a la problemática, revaluar cada una de sus causas, rediagnosticar, y aplicar remedios efectivos. ¿Y cómo lo hacemos? Primero entendiendo por qué fracasó la supuesta reforma del sistema de inversión pública. En nuestra opinión se debe a una visión y dimensionamiento limitado del problema. Es decir, que los policy makers concibieron el problema únicamente al interior del sistema, sin considerar el carácter integral, transversal e intersistémico que tiene con otros, tales como los sistemas de planificación, presupuesto, tesorería, endeudamiento, contratación pública, recursos humanos y control gubernamental.

En otras palabras, pretender una mejora del sistema de inversión pública creyendo que el problema radica únicamente en las normas, procedimientos, directivas, entre otros que emite el órgano rector de dicho sistema, supone caer en un razonamiento falaz que podría explicarse por el conocimiento que se tiene de la cosa pública, lo que trae como consecuencia — como en efecto ha ocurrido- un deterioro sostenido de la inversión pública en el país en los últimos cinco años.

En efecto, para que un proyecto de inversión pública pueda ejecutarse debe seguir pasos tanto al interior del sistema de inversión pública como fuera de él. De hecho, se inicia fuera de él cuando el Estado, luego de una evaluación de brechas de infraestructura o de servicios pú- 
blicos, establece los proyectos de inversión que requiere realizar en un período determinado. Es decir, se inicia (o debería iniciar) con el sistema de planificación. Luego, recién debe pasar por el sistema de inversión pública, iniciando la etapa de preinversión. En esta fase, sí se requieren ajustes importantes con la finalidad de fortalecer la optimización y dinamizar la elaboración de los proyectos. Así, sería deseable que se estandarización de los estudios de preinversión, sobre todo, en aquellos proyectos que son recurrentes, como los colegios, los establecimientos de salud en todos sus niveles (I, II y III), comisarías, pistas y veredas, entre otros.

En otras palabras, si bien el invierte.pe trajo consigo la creación de fichas técnicas para proyectos estándar, los cuales traen consigo datos pre cargados para las principales variables para el dimensionamiento y costos de un proyecto, en la realidad no han alcanzado el objetivo propuesto, por lo que deberían revisarse las fichas estandarizadas, con mayor énfasis en proyectos que reúnen características tales como: (i) alcance nacional, (ii) rápido impacto, (iii) genere empleo directo; $y$, (iv) cierre brechas de infraestructura y de servicio público.

En efecto, el sistema de inversión pública debe tener ex ante la preinversión pre-elaborada para este tipo de proyectos, solo para completar información ajustada a la demanda de los servicios en la zona, entre otros factores que no impliquen un nivel de complejidad mayúsculo, en términos de esfuerzo, tiempo y recursos.

Para esto sí es necesario una modificación para estandarizar las fases de formulación. Se han hecho algunos esfuerzos en materia educativa, a través del PRONIED (Programa Nacional de Infraestructura Educativa), y, en materia de salud, el PRONIS (Programa Nacional de
Inversiones en Salud) ha intentado hacer lo propio, aunque sin mucho éxito, ya que depende normativamente del Ministerio de Salud, donde cambiar un procedimiento puede ser una tarea tan titánica como lograr expropiar los terrenos para construir la línea 2 del Metro de Lima. Conseguir estandarizar este tipo de proyectos permitirá dinamizar rápidamente la economía al generar empleo directo y descentralizado a lo largo de todo el territorio nacional, generando impacto importante en el crecimiento de la economía nacional y local.

No obstante, una vez que el proyecto es viable, puede empezar recién un vía crucis, tanto por la asignación de recursos como por el inicio del proceso de licitación. Es decir, nos encontramos frente a dos sistemas administrativos orientados a obtener financiamiento, que puede ser con recursos del tesoro público (sistema de presupuesto público) o con alguna operación de endeudamiento (sistema de endeudamiento público).

Una vez obtenido el financiamiento para el proyecto, se debe buscar a la persona, natural o jurídica, que se encargue de ejecutar el proyecto de inversión. Entonces, nos introducimos a otro sistema administrativo, que es el de abastecimiento, dentro del cual se encuentra la contratación pública. Es decir, ingresamos a un sistema que también enfrenta una serie de desafíos, tanto por el tiempo que implica obtener al proveedor, como por lograr que sea de calidad y cumpla con los cronogramas de ejecución previstos inicialmente.

Actualmente, se está trabajando una nueva ley de contrataciones. Sería la tercera ley en los últimos 13 años; y en los últimos cinco años se ha cambiado dos veces. En promedio se cambia íntegramente la ley cada cinco 
ańos, lo que claramente genera marchas y contramarchas, cuyos efectos son percibidos de manera más sensible por la inversión pública.

Para todo ello, se requiere de personal calificado y eficiente que permita alcanzar los resultados previstos dentro de los cronogramas establecidos, para lo cual, debemos ingresar a otro sistema administrativo: el de recursos humanos. Pese a que se ha generado una importante reforma en el sistema del servicio civil, el cual, lamentablemente, ha tenido poco apoyo político en el último quinquenio para su implementación (salvo en el periodo del ministro Carlos Oliva), lo que ha generado es que luego de ocho años (supuestamente la fase final de la implementación progresiva), no exista una sola entidad del gobierno nacional que haya implementado íntegramente la ley y ya cuente con todos sus directivos bajo el régimen del servicio civil.

Entre las ventajas que genera el régimen del servicio civil para la administración pública, se encuentran la línea de carrera, enfoque de servicio en el ciudadano, gestión por resultados, evaluaciones anuales en función de metas, objetivos e indicadores claramente establecidos, capacitación al personal de manera permanente, identificación de oportunidades de mejora para el personal que no haya tenido una evaluación positiva, entre otras. Si esta ley estuviera ya implementada, los niveles de ejecución registrarían cifras mucho más alentadoras, dados los incentivos cruzados que conlleva.

Finalmente, uno de los sistemas administrativos transversales a todas las fases por la que discurre un proyecto de inversión pública y que ha sido también motivo de retrasos en la ejecución de proyectos, es el control gubernamental. Y es que ante un problema administrativo o, incluso, indicios de delito, termina afectán- dose el proyecto más que el o los agentes que supuestamente delinquieron, lo que genera un círculo vicioso, pues afecta en múltiples dimensiones a la sociedad: crecimiento de la economía, empleo, ocasionaa sobrecostos por los gastos por servicios generales que se generan con la paralización de una obra, postergación del servicio público que se iba brindar en la infraestructura paralizada, entre otros.

Entonces, se deben generar esquemas que permitan aislar el proyecto de los actos ilícitos, sean estos administrativos o penales, de tal manera que, ante un acto ilícito, se sancione a los responsables sin que se vea afectada toda la cadena de pagos asociada. Parece que la frondosa evidencia generada por el club de la construcción y Lava Jato no ha suficiente para que se considere una alternativa para lo señalado.

En ese orden de ideas, concebir como único problema de la ejecución de la inversión pública al sistema que lo regula y solo intervenir en él, resulta insuficiente, ya que es un sistema que actúa - como ya hemos referido-intersistémicamente, y que nos lleva a la necesidad de realizar un análisis holístico e intervenciones que permitan agilizar las diferentes fases de los sistemas administrativos por el que debe transitar un proyecto de inversión pública, desde su ideación hasta su puesta en operación.

\section{SOBRE LOS SISTEMAS ADMINISTRA- TIVOS INTERVINIENTES EN LA EJECU- CIÓN DE UN PROYECTO DE INVERSIÓN PÚBLICA}

\subsection{Sistema de Planificación}

El Perú cuenta con el Sistema Nacional de Planeamiento Estratégico (Sinaplan), siendo su 
órgano rector el Centro Nacional de Planeamiento Estratégico (Ceplan), ambos creados mediante el Decreto Legislativo N. 1088 en el 2008.

El Sinaplan es un conjunto articulado e integrado de órganos, subsistemas y relaciones funcionales cuya finalidad es coordinar y viabilizar el proceso de planeamiento estratégico nacional para promover y orientar el desarrollo armónico y sostenido del país. Entre los objetivos del Sinaplan, destaca su función de constituirse como el espacio institucionalizado para la definición concertada de una visión de futuro. Asimismo, debe articular e integrar en forma coherente y concertada las diferentes propuestas y opiniones para la elaboración del Plan Estratégico de Desarrollo Nacional y los planes nacionales, sectoriales, institucionales y subnacionales, así como las orientaciones, los métodos, los procesos y los instrumentos para el planeamiento estratégico.

Se señala que el monitoreo de la gestión tiene un enfoque orientado a resultados, siendo el Ceplan el encargado del seguimiento y evaluación de planes, políticas, programas, objetivos y proyectos, con posibilidad de emitir informes periódicos a solicitud del presidente de la República o del presidente del Consejo de Ministros, sobre la situación económica, social, ambiental e institucional del país.

Se establece que los objetivos estratégicos del Plan Estratégico de Desarrollo Nacional orientan la formulación de las políticas nacionales, que se traducen en las políticas del Gráfico 5.

Gráfico 5. Políticas de desarrollo nacional

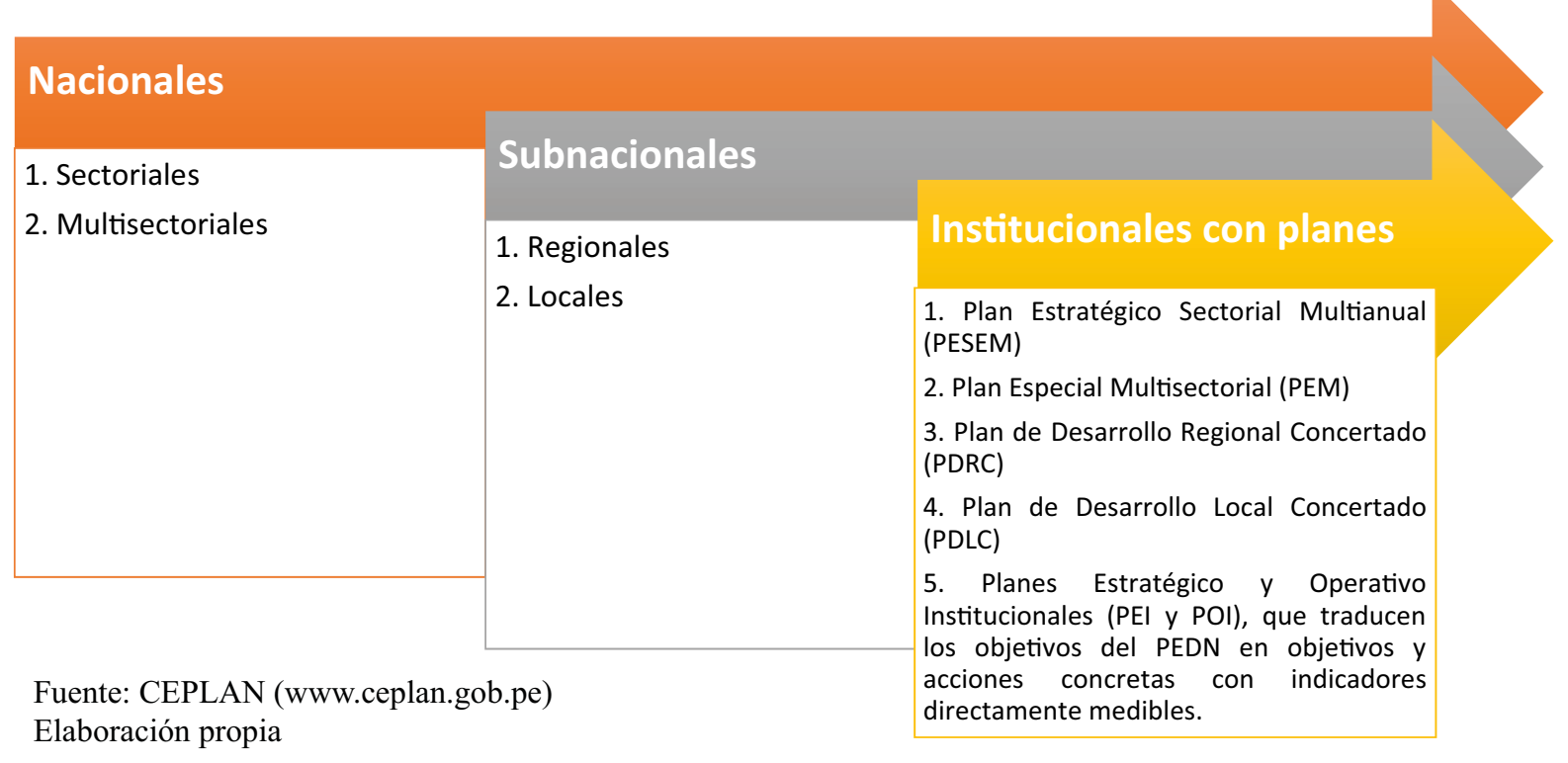

Así, podríamos seguir señalando aspectos que en el papel responden a un modelo ideal, pero que, en realidad, es solo una utopía. Los planes en la realidad están plagados de acciones generales y, en muchos de los casos, no tienen una vincula- ción directa con el presupuesto (salvo formalmente). Es por ello que en vez de cerrar brechas de infraestructura y de servicios públicos básicos para los ciudadanos, estas se van ampliando. 
Si todas las entidades aprueban periódicamente los diferentes planes y políticas señaladas en líneas precedentes, y estos se encuentran orientados — al menos teóricamente- a alcanzar resultados concretos, nos surge la interrogante de por qué no mejoran los servicios al ciudadano, la institucionalidad ni la calidad de los servicios públicos. Algo está sucediendo que impide que el común de los ciudadanos puedan apreciar los «grandes resultados» de dichos planes.

Por ello, deben revaluarse los indicadores que se utilizan y reorientarlos a resultados concretos, tangibles y medibles que generen un cambio real en la vida de los ciudadanos, y evitar caer en la complacencia de cumplir formalmente todas las evaluaciones traducidas en la verificación del cumplimiento de actividades, no de metas, es decir, quedan en el papel con cero impacto positivo en la población, a quienes finalmente se debe el Estado para asegurar la provisión de servicios básicos como agua, luz, salud, educación o justicia.

Ahora bien, tal vez uno de los planes más importante es el Plan Nacional de Infraestructura para la Competitividad, conocido como el PNIC, que constituye el instrumento más importante que obliga al Estado a disciplinarse y mantener un orden, tanto fiscal como de cierre de brechas, lo que permite una programación ordenada en el mediano y largo plazo, al mismo tiempo que se fortalece la institucionalidad, ya que, independientemente de quién sea el ministro o jefe de la entidad, los proyectos seguirán un procedimiento de incorporación en el PNIC si se quiere que sean financiados.

\subsection{El Sistema Nacional de Programación Multianual y Gestión de Inversiones (invierte.pe)}

Este sistema, que reemplazó al tradicional Sistema Nacional de Inversión Pública (SNIP), fue creado el $1 \mathrm{de}$ diciembre de 2016, mediante el Decreto Legislativo N. ${ }^{\circ} 1252$, y entró en vigencia el 15 de febrero del año 2017, un día después de la publicación de su reglamento.

Este nuevo sistema buscaba que la inversión pública sea destinada de manera inteligente, es decir, que ayude a reducir brechas, a elaborar adecuadamente los estudios de preinversión, a reducir los tiempos para formular y evaluar y al seguimiento de las obras realizadas. Los nuevos cambios introducidos por invierte.pe —al menos teóricamente- mejoran el ciclo de inversiones, el cual está compuesto por cuatro fases (Gráfico 6).

Gráfico 6. Ciclo de inversiones

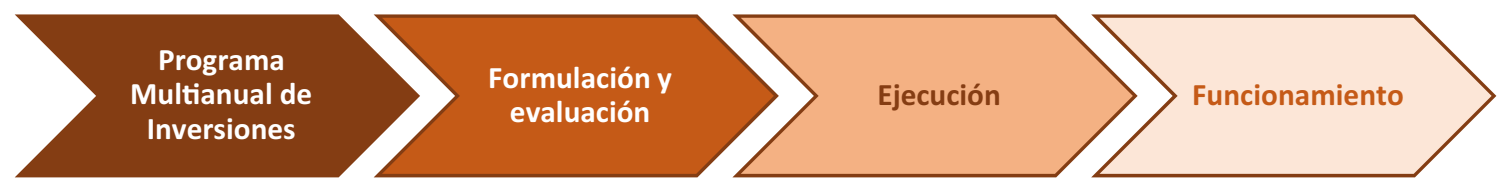

a) Programación Multianual de Inversiones (PMI)

Dentro de esta programación es importante:

(i) definir los indicadores de brechas (sobre la base de un diagnóstico ${ }^{1}$, y (ii) realizar la

1 Cada sector, a través de su OPMI (antes OPI), debe elaborar un diagnóstico de brechas de infraestructura y servicios públicos, en función a este diagnóstico se establecen los objetivos para reducir las brechas, por áreas geográficas, que comunican a los GR y GL. 
Gráfico 7. Directivas para Proyecto de Inversión Pública (PIP)

\begin{abstract}
Recuadro 1.
Para incorporar un Proyecto de Inversión Pública (PIP) después de que la PMI ha sido aprobada, la OPMI (antes OPI) deberá justificar el pedido y este deberá ser aprobado por el Órgano Resolutivo (OR) correspondiente (titular del sector, gobernador o alcalde). De no contar con marco presupuestal, no pasará a la siguiente fase. Posteriormente, se debe establecer la cartera de inversiones, las OPMI del sector y de los GR y GL elaboran su cartera de inversiones sobre la base de los objetivos y priorización definidos.

El PMIE se consolida cuando el Órgano Resolutivo de cada sector, GR y GL presentan, antes del 30 de marzo de cada año, su PMI a la Dirección General de Presupuesto Multianual de Inversiones (DGPMI), que consolida toda la información en el Programa Multianual de Inversiones del Estado (PMIE). Si bien el PMIE es el marco de referencia de la inversión pública, previamente es evaluado por la DGPMI para validar su consistencia con la programación de inversiones que realizan anualmente los sectores, GR y GL, y cuya información es remitida por la Dirección General de Presupuesto Público.
\end{abstract}

Fuente: Benites y Garcés (2012)

programación multianual (mínimo 3 años) en base a las brechas, estándares de servicio y niveles de producción ${ }^{2}$. La cartera de inversiones debe indicar la posible fuente de financiamiento, la modalidad de ejecución, el monto referencial y las fechas de inicio y término. En esta primera fase, el principal cambio es la definición de proyectos, no todas las inversiones son consideradas PIP, y esto permite focalizar el invierte.pe en obras de importancia ${ }^{3}$ (Gráfico 7).

2 Las OPMI de cada GR y GL elaboran sus PMI y se lo comunican a cada sector.

3 Se califica como PIP a la formación de capital físico, humano, natural, institucional o intelectual que tenga como propósito crear, ampliar, mejorar o recuperar la capacidad de producción de bienes o servicios que el Estado tenga responsabilidad de brindar o de garantizar su prestación. Se califica como no PIP a la optimización (compra de terrenos e inversiones menores que resultan de hacer un mejor uso de la oferta existente), ampliación marginal (incrementan el activo no financiero de una entidad pública pero que no modifican su capacidad de producción de servicios o, que de hacerlo, no supera el $20 \%$ de dicha capacidad en proyectos estándar), reposición (reemplazo de activos que han superado su vida útil), rehabilitación: (reparación o renovación de las instalaciones, equipamiento y elementos constructivos sin ampliar la capacidad de provisión de servicios).

\section{b) Formulación y evaluación}

En esta etapa se elaboran las fichas técnicas o estudios de preinversión, así como también se evaluará y registrará el proyecto en el banco de inversiones. La innovación insertada por invierte.pe en esta etapa es la creación de fichas técnicas para proyectos estándar, las cuales son formatos estandarizados precargados con datos para las principales variables para el dimensionamiento y costos de un proyecto. Estas fichas brindan una justificación técnica y económica clara y concisa. La OPMI sectorial es la encargada de recomendar aquellas tipologías de proyectos que pueden estandarizarse, los proyectos no estandarizables requerirán estudios de preinversión. Inicialmente se han identificado 51 tipos de inversiones estándar (entre $70 \%$ y $80 \%$ del presupuesto de inversión que evaluaba el SNIP).

\section{c) Ejecución}

La Unidad Ejecutora de Inversiones (UEI) elaborará la ficha técnica en función de la 
Tabla 1. Órganos de Invierte.pe

\section{Sector / gobierno regional/ gobierno local}

- Órgano Resolutivo (OR)

- Oficina de Programación Multianual de Inversiones (OPMI)

- Unidades Formuladoras (UF)

- Unidades Ejecutoras de Inversiones (UEI)

concepción técnica y dimensionamiento del estudio de preinversión. En el caso de las inversiones que no constituyen PIP, se elabora un informe técnico sobre la base de la información registrada directamente en el Banco de Inversiones. El principal cambio implementado en esta etapa es que el seguimiento de la ejecución se realiza a través de Sistema de Seguimiento de Inversiones, herramienta que asocia el Banco de Inversiones con el SIAF (Sistema Integrado de Administración Financiera). Si se realizan modificaciones, la UEI o UF, según corresponda, debe registrarlas en el Banco de Inversiones antes de ejecutarlas. Culminada la ejecución, la UEI realiza la liquidación física y financiera y cierra el registro en el Banco de Inversiones.

\section{d) Funcionamiento (Tabla 1)}

El nuevo cambio en esta fase es la creación de reportes anuales de activos, los titulares de los activos incluidos en el PIM deben reportar anualmente su estado a las OPMI del sector, GR o GL, respectivamente. Asimismo, deben programar el gasto necesario para asegurar la operación y mantenimiento de los mismos. La evaluación ex post de los proyectos está a cargo de la DGPMI, que tiene la facultad de publicar el listado de proyectos

\section{Ministerio de Economía y Finanzas}

- Dirección General de Programación Multianual de Inversiones (DGPMI):

- Dirección General de Presupuesto Público

- Dirección General de Endeudamiento y Tesoro Público.

que deberían ser evaluados cada año por las OPMI del sector, GR o GL, estableciendo previamente los criterios de evaluación.

\section{Características del sistema}

El Sistema Nacional de Inversión Pública del Perú, al menos en el papel, se caracteriza por:

i) Eficiencia. La misma que es influenciada, al menos formalmente, por su mayor asistencia técnica en la etapa de preinversión, el desarrollo de capacidades en todos los niveles de gobierno, la programación estratégica de la inversión pública y su seguimiento y la mejora de procesos para evitar trabas burocráticas.

ii) Transparencia. La información es pública, lo que le permite monitorear la ejecución del gasto, de manera agregada y a nivel de detalle.

iii) Institucionalidad. Cuenta con un marco normativo para la inversión pública, un órgano rector, así como órganos específicos al interior de las entidades públicas.

iv)Tecnicismo. Cuenta con directivas y guías metodológicas que reducen la discrecionalidad en la formulación y evaluación, sobre 
todo en la etapa de preinversión, definiendo contenidos mínimos, parámetros y normas técnicas, parámetros de evaluación, pautas para verificación de viabilidad, entre otros documentos normativos.

La interrogante que surge es la siguiente: ¡̨ómo es que, a pesar de tener un marco normativo eficiente, los resultados de la inversión pública en el último quinquenio muestran un marcado deterioro respecto del quinquenio previo? La respuesta parece estar orientada a un problema de personas y no legal. Es decir, lo que estaría fallando es la gestión pública, los gestores públicos, los operadores públicos encargados de materializar las grandes líneas maestras diseñadas en las normas legales vigentes.

Entonces, un proyecto de inversión pública, una vez que ha sido formulado y que ya tiene viabilidad, estaría expedito para la siguiente fase, que consiste en cómo financiarlo, y, para ello, nos introducimos a dos sistemas administrativos: (i) El presupuesto público, donde podremos determinar si es que existen recursos disponibles por la fuente de financiamiento recursos ordinarios (impuestos) y (ii) endeudamiento, sea externo o interno, dependiendo de los costos del proyecto.

\subsection{Financiamiento de los proyectos: Siste- ma de Presupuesto y Endeudamiento}

Una vez que los proyectos obtienen la viabilidad en el marco del invierte.pe, el siguiente paso es obtener el financiamiento. En este caso, dependerá del nivel de envergadura del proyecto, el alcance, número de beneficiarios, monto de inversión y espacio fiscal de la entidad, para determinar la forma cómo se financiará.

Si se financiará con recursos ordinarios, es decir, del tesoro público, entonces la entidad deberá incluir dicho proyecto dentro de su programación presupuestal, dentro del espacio fiscal existente para lograr el financiamiento. Si la entidad no tuviera espacio, y tampoco tuviera recursos determinados (provenientes de la explotación de los recursos naturales), entonces lo más probable es que requiera endeudarse. Para ello, habría que seguir todo el proceso de concertación de operaciones de endeudamiento. Debe definirse si habrá contrapartida y de cuánto será. Asimismo, precisar si será interno o externo.

En este caso, deberá seguir el proceso natural de concertación que, si se tratase de un endeudamiento externo, tendría que solicitarse autorización al consejo de ministros para iniciar negociaciones con algún organismo multilateral, como el Banco Mundial, Banco Interamericano de Desarrollo, el Banco de Desarrollo de América Latina, Fondo Monetario Internacional, entre otros. Una vez que se reciba dicha autorización, el MEF en su calidad de agente financiero del Estado es el encargado de llevar a cabo el proceso de concertación. Para ello, existen una serie de requisitos y condiciones que debe cumplir la entidad que requiere el financiamiento. Incorporar en el Plan Anual de Concertaciones y luego regresar al Consejo de Ministros, previa opinión favorable de la Contraloría General de la República, para la aprobación de la operación.

Evidentemente, financiar un proyecto con endeudamiento implicará un consumo mayor de tiempo, dado los diferentes pasos que involucra. Por eso, no debe soslayarse ese elemento dentro del cronograma.

En el caso de proyectos que requieran financiamiento durante el ejercicio presupuestario, la entidad debe solicitar al MEF la disponibilidad de recursos. Este evaluará, luego de moni- 
torear el ritmo de ejecución de gasto, si existe posibilidad de hacerlo, buscando incluir en el presupuesto del siguiente ejercicio fiscal, los montos que se ejecutarán.

Entonces, en los sistemas administrativos vinculados al financiamiento, deben seguirse las fases, tanto de programación y ejecución presupuestal, como de programación, concertación y aprobación de operaciones de endeudamiento. En estos sistemas administrativos, específicamente, de la administración financiera del Estado, no existe mucho espacio para incorporar mejoras adicionales a las generadas en el año 2018.

Con una adecuada planificación, la programación del financiamiento de los proyectos sería mucho más ordenado y se optimizarían los tiempos de atención de los recursos requeridos.

\subsection{Sistema de Abastecimiento: contrata- ción pública}

Culminado el vía crucis de la formulación y de la obtención de financiamiento para el proyecto de inversión pública, empieza un largo camino por recorrer. Nos referimos a la contratación del proveedor que ejecutará el proyecto de inversión. Y es que los distintos procesos de contratación tienen varias fases que demandan un nivel de conocimiento y de experiencia que eviten que los procesos queden desiertos o que minimicen el riesgo de impugnación por falta de claridad, ambigüedad o restricciones a la competencia en las bases. Todo ello puede llevar a dos grandes problemas cuando una entidad requiere ejecutar un proyecto de inversión pública: (i) que el proceso sea declarado desierto, o (ii) que la adjudicación de la buena pro sea impugnada.
Sobre el primer punto, existen cuatro grandes razones que pueden generar esa declaratoria de desierto: (i) No se registraron participantes, (ii) se registraron participantes, pero no presentaron ofertas, (iii) se presentaron ofertas, pero estas no fueron admitidas; $y$, (iv) Se adjudicó, pero no se firmó el contrato por razones diversas.

Por ejemplo, el OSCE (Organismo Supervisor de las Contrataciones del Estado), en un estudio realizado en el 2011, advirtió que un $15 \%$ en promedio de los procesos de selección son declarados desiertos. Entre las causas que pueden explicar el número de desiertos, este organismo encontró que el $71 \%$ tiene como causales comunes la existencia de diversos aspectos de las bases administrativas que representaron barreras, principalmente relacionadas con el cumplimiento de los requerimientos técnicos mínimos y los factores de evaluación. Es decir, un factor importante es la definición de lo que la entidad necesita, pues, de este modo, se podrá delinear adecuadamente los requisitos técnicos mínimos. De tal manera que no se exija más de lo que se necesita ni se pida menos de lo que se requiere.

Si bien el OSCE no descarta que una propuesta técnica que termine invalidándose debido a la falta de pericia y/o capacitación de los proveedores para formularla, consideramos que ello es un elemento que la entidad respectiva puede mitigar; ya que si esta hubiera hecho un estudio de mercado adecuado entre los potenciales postores que pueden cumplir con el objeto de contratación, teóricamente serían postores con experiencia participando de este tipo de procesos. Por lo que, en nuestra opinión sigue estando en cabeza de la entidad el adecuado diseño de las bases, al ser formuladas luego de una indagación del mercado. 
Ahora bien, ese $75 \%$ se descompone de la siguiente manera: (i) un $45 \%$ de desiertos se debe a la inexistencia de propuestas válidas, es decir, no hubo participantes o existiendo, sus propuestas fueron descalificadas. Y el $26 \%$ restante, se debió a que los postores no alcanzaron el puntaje mínimo. Es decir, cumplieron con los requisitos de las bases, pero no les alcanzó para el puntaje mínimo requerido por las bases. En cualquier caso, son las bases las que finalmente permiten que haya o no postores válidos, sea porque son demasiado exigentes o que esa exigencia mayor no se traduce en los valores referenciales (hoy no publicitados), es decir, los postores ofertan por encima del valor estimado determinado por la entidad.

Por otro lado, si bien el número de impugnaciones presentadas contra las adjudicaciones de la buena pro están fuera del control de la entidad, la forma de mitigarlos sí está bajo su control. Es decir, si, por ejemplo, las bases restringieran la competencia o tuvieran una especificación técnica ambigua, entonces, el riesgo futuro de impugnación será más alto, respecto de especificaciones técnicas contenidas en las bases que estuvieran bien definidas y formuladas.

En efecto, la Ley de Contrataciones ya ha establecido que para impugnar se requiere la presentación de una carta fianza equivalente al 3 $\%$. Entonces, esa garantía supone que el postor que decide impugnar debe verificar que existan elementos que justifiquen la presentación de un recurso, es decir, si existieran bases ambiguas o que restringen competencia, este tendrá un argumento para iniciar el procedimiento recursal con las expectativas, no menor, de obtener un resultado favorable; no obstante, ser responsabilidad absoluta de la entidad contratante.

Uno de los sistemas administrativos que representan un cuello de botella para la ejecución de los proyectos de inversión es precisamente el de abastecimiento, específicamente, el de contrataciones del Estado. Por ello, se requiere realizar modificaciones, pero no necesariamente a la normatividad de contrataciones, sino más bien a un trabajo más intenso del OSCE y del MEF en capacitar a las entidades del sector público para fortalecer las capacidades de los órganos encargados de las contrataciones, así como de las áreas usuarias.

\subsection{Sistema de Tesorería}

Recordemos que para que se considere ejecutado un proyecto, desde el punto de vista presupuestal, este debe seguir todas las fases, es decir: (i) certificado, (ii) comprometido, (iii) devengado, (iv) girado; y (v) pago.

Antes de iniciar el proceso de selección, se requiere tener la certificación presupuestal. Luego de adjudicar la buena pro y firmado el contrato, se afecta presupuestalmente a través del compromiso. Una vez ejecutado el contrato, y con la conformidad del área usuaria, la afectación presupuestal se denomina devengado. Y, finalmente, se inicia el proceso de giro para el pago. Este último tramo, el girado, se encuentra a cargo de otro sistema administrativo: el de tesorería.

Teóricamente, es una fase en la que solo debería seguir un procedimiento de carácter formal y de verificación de datos e información. No analizan el contenido, pues eso es responsabilidad del área usuaria. No obstante, esta es una fase que no es del todo célere, a pesar de las modificaciones legislativas orientadas a ese fin. Así, la última modificación al reglamento de la ley de contrataciones se redujo de 15 a 10 días calendario, realizada a fines de junio del 2020. 
A pesar de eso, pareciera que aún sigue siendo mucho tiempo para que se dé cumplimiento a la parte menos compleja de toda la cadena. Esta fase, aunque importante, es más de tipo operativo, por lo que, mejorando los procesos, incorporando tecnologías de la información, así como innovación y desarrollo, se pueden acelerar, todavía más, dichos plazos. En algunos casos puede demorar entre 15 y 30 días, en promedio. Pero pueden llegar a tardar, incluso, más de un mes.

En este caso, no es que la regulación del sistema administrativo de tesorería sea complejo o burocrático. En este punto nos encontramos frente a un problema de gestión o gerenciamiento pública, es decir, de la capacidad de las personas para agilizar dichos pagos.

Si bien para este momento, desde el punto de vista presupuestal, ya se considera "ejecutado" el gasto, en realidad la ejecución financiera es relevante por el costo financiero que puede generar a los proveedores, sobre todo si se trata de MYPES. A pesar de varios esfuerzos de regular consecuencias jurídicas a dichas demoras, en la realidad, existen aún entidades que dilatan esta fase, con lo cual no se logra generar el dinamismo económico en los términos definidos en el diseño normativo.

En efecto, la parte más compleja, en el caso de obras, sobre todo, es para el proveedor lograr la conformidad de obra, pues es la parte medular respecto de la contratación realizada. En esta fase suelen existir observaciones por parte de la entidad, que luego deben ser levantadas por el contratista. Esta fase, sin duda, tiene un tiempo - a veces- no menor, pero que en algunos casos se compara con el tiempo para realizar el pago.
En ese sentido, se debe incorporar tecnología, mejorar los procesos y fortalecer el capital humano que permita optimizar y agilizar la fase de pago a las empresas contratistas del Estado, que contribuyan a la dinamización de la economía.

\subsection{Sistema de Recursos Humanos}

Este sistema administrativo, a diferencia de los previamente comentados, es transversal, es decir, se requiere capital humano para realizar buenos planes, que puedan establecer indicadores de evaluación adecuados, que se encuentren en capacidad de realizar evaluaciones que permitan mejorar tanto las capacidades como los procesos involucrados.

De igual modo, para realizar los estudios de preinversión se requiere de personal idóneo, con conocimientos sólidos que permitan formular proyectos de inversión adecuado. Para estandarizar los proyectos en educación, salud, seguridad, entre otros, se requiere de personas capacitadas.

Por su parte, para ejecutar los proyectos de inversión es necesario contar con servidores y funcionarios que conozcan la ley de contrataciones. No solo en las oficinas encargadas de la contratación, sino en toda la entidad, ya que es a partir de estas que se inician los procesos de contratación, a través del "requerimiento".

Dicho requerimiento es formulado por el área usuaria. Dentro de esta, por lo general, lo realiza el asistente administrativo. Muchas veces surgen errores en su elaboración, que no son identificados por el jefe del área, y cuando es remitido al órgano encargado de las contrataciones, este tampoco los advierte, ocurriendo lo mismo cuando lo revisa el comité especial. Los recursos humanos son clave en esta tarea. 
De igual modo, el área usuaria es la encargada de mantener la relación con el contratista y debe estar en la capacidad de ser una contraparte idónea, tanto para advertir omisiones o errores de los contratistas como para observar aspectos que no son técnicamente correctos, dilatando innecesariamente la ejecución del proyecto. Al mismo tiempo, una vez otorgada la conformidad, se requiere celeridad para realizar los pagos a los contratistas, con la finalidad de dinamizar con mayor rapidez la economía, dando impulso importante a la demanda. Para ello, se requiere fortalecer también a los equipos que trabajan en las oficinas de administración, dentro de las cuales se encuentra las oficinas de contabilidad y tesorería, áreas clave para materializar los pagos a las empresas que obtuvieron la conformidad del servicio o de obra.

En ese contexto, implementar la reforma del servicio civil aprobada hace casi 10 años resulta indispensable si es que realmente se pretende agilizar la ejecución de los proyectos de inversión y, más aún, si se planea dar un fuerte impulso a través de un shock de la inversión pública, ya que, como hemos señalado en el presente trabajo, sería un error considerar cualquier reforma como si se tratara de un compartimento estanco, cuando en realidad debe dársele un enfoque holístico, que involucra a todos los sistemas administrativos que han sido materia de análisis.

\subsection{Sistema Nacional de Control}

Finalmente, otro de los sistemas administrativos que funcionan de manera transversal y que deben contribuir a que las entidades públicas hagan un uso adecuado de los recursos públicos, es el Sistema Nacional de Control, que a diferencia de los otros sistemas materia de aná- lisis, se encuentra a cargo de una entidad con rango constitucional.

La autonomía constitucional le permite realizar su labor con absoluta independencia y autonomía del poder político, ya que, el control gubernamental es clave para reducir los espacios de corrupción en las entidades públicas. Para ello, existen condiciones que permiten reducir esos espacios. Las buenas prácticas internacionales señalan que debe ser un órgano con autonomía constitucional, es decir, que no esté sometido jerárquicamente a ningún otro poder del Estado. Asimismo, es deseable que su duración en funciones trascienda al gobierno de turno, que sea elegido por un órgano distinto al del Ejecutivo y que tenga autonomía económica.

Finalmente, en cuanto a la independencia administrativa de las unidades de control, es importante la independencia que tienen los jefes de las unidades de control al interior de cada entidad. En el caso del Perú, los jefes de los órganos de control institucional, es decir, los jefes de las oficinas de control alojadas al interior de las entidades, dependen administrativamente de la Contraloría General de la República, por lo que ésta es su empleadora directa, y no la entidad en la cual despliegan sus funciones de control.

Al respecto, podemos afirmar que la arquitectura legal peruana cuenta con sólidas bases comparada con los países centroamericanos, por ejemplo. La mayoría de los países, salvo Nicaragua, los jefes de las unidades de control solo tienen dependencia funcional respecto de la Contraloría, existiendo dependencia administrativa entre la entidad auditada y el jefe de los auditores en esa entidad, lo que incrementa el riesgo que los titulares de las entidades puedan ejercer algún grado de injerencia sobre el jefe del órgano de control en su entidad. 
Ahora bien, en la realidad peruana, esa independencia administrativa no se ve en todas las oficinas de control institucional, ya que muchos de los trabajadores forman parte de la planilla de la entidad auditada y no de la Contraloría General de la República (CGR). Es más, existen algunas entidades, con poco presupuesto, que no cuentan con una oficina de control institucional. Debe fortalecerse el control a nivel nacional y generar lecciones aprendidas desde la CGR que le permitan implementarlas para prevenir actos ilícitos previamente advertidos o identificados.

El cambio de enfoque de la CGR es fundamental. En realidad, la idea de que mientras más hallazgos encuentra un auditor es mejor auditor, es una mentalidad que debe ser desterrada. La CGR debe, a partir de las experiencias previas, adelantarse y advertir a los titulares de las entidades de las acciones que están observando, y que en el pasado tuvieron un resultado determinado que afectó los recursos públicos, la operatividad o el servicio que prestan al ciudadano. Es decir, cumplir una labor preventiva.

Pero un tema que también resulta clave y que no puede soslayar la CGR es el capital humano, es decir, debe contar con personal capacitado sólidamente en cada uno de los temas que le toque auditar y en la especialidad requerida. En el pasado ha ocurrido que contadores emitían juicios sobre temas legales de alta especialización, $\mathrm{y}$ abogados, juicios sobre temas ingenieriles. Claramente este tipo de hallazgos suscritos por personal que no está formado en la materia que se está analizando debilita su accionar.

Ahora estos son los casos más extremos. Existen otros al interior de la propia profesión, pero con distinta especialidad. Por ejemplo, que un abogado con especialización en dere- cho civil y con experiencia en el sector privado analice contratos administrativos en materia de contrataciones del Estado. $\mathrm{O}$ un abogado con especialización en derecho administrativo, pero sin conocimientos de inversión pública.

En ese sentido, la CGR debe actuar ex ante y ex post. Debemos fortalecer el accionar de la Contraloría para evitar más clubes de la construcción. ¿El ministerio de Transportes será el único club? ¿Durante tantos años la CGR no analizó los sobreprecios? Tal vez porque no tenían economistas y abogados con especialización en competencia o en regulación. La CGR es la primera entidad que tiene acceso a toda la información disponible que, además, puede apoyarse en otras entidades, como, por ejemplo, el Indecopi.

En las últimas semanas, la Comisión de Defensa de la Libre Competencia del Indecopi sancionó con 25 millones de soles a un grupo de empresas que se coludieron y se repartieron los procesos de selección convocados por el Ministerio de Educación y uno del INEI. El período de colusión fue entre el año 2009 y el 2016, es decir, siete años. ¿Qué pasó con la CGR? ¿Por qué no se activaron las alertas tempranas? Ya hemos visto que pasó en el MTC, ahora en el Minedu. ¿¿Habrá alguna otra entidad? ¿La CGR ha identificado patrones de conducta que pueden haberse reproducido en alguna otra entidad? Y el caso de educación no fue en materia de infraestructura, pero tal vez pueda existir también. Habría que revisar. Tendría que haber una labor un poco más proactiva.

Ahora, lo que contribuyó ciertamente a que el Indecopi pueda descubrir este cartel de editoriales fue el Programa de Clemencia, implementado desde hace no muchos ańos y que requiere un fortalecimiento mayor para descubrir a más empresas que no creen en el libre 
mercado y buscan maximizar su beneficio de manera ilícita, a través de la colusión.

En ese orden de ideas, la CGR debe estar en permanente fortalecimiento de capacidades a su personal, al mismo tiempo debe incorporar medios informáticos y estandarizar y capacitar a los funcionarios de manera preventiva.

\section{LOS CONTRATOS GOBIERNO A GOBIERNO COMO ALTERNATIVA A LA PROBLEMÁTICA DE LA EJECUCIÓN DE LA INVERSIÓN PÚBLICA. ¿UNA BUENA SOLUCIÓN?}

En los últimos cuatro años, el nivel de ejecución de los proyectos de inversión pública ha venido cayendo de manera sostenida por diversos factores, tanto estructurales y transversales como coyunturales. Dentro de los estructurales y transversales tenemos, a su vez, dos grandes factores: por un lado, la política no escrita orientada a paralizar (o ralentizar) la implementación de la ley del servicio civil, la cual está orientada a fortalecer el capital humano en las entidades públicas, al ser este la piedra angular para la implementación de cualquier política pública, incluida la ejecución de los proyectos de inversión; y por otro, los cambios realizados al entonces sistema nacional de inversión pública por el nuevo invierte.pe, que no es otra cosa que el SNIP remozado. Es decir, como ya lo hemos señalado, sin un equipo técnico calificado al interior del Estado, en todos sus niveles, y con un sistema de inversión pública con cambios cosméticos, se convierte en un escenario perfecto para alcanzar los niveles de ejecución de la inversión vistas últimamente.

Dentro de los factores coyunturales se encuentra el factor político, particularmente, las elecciones subnacionales realizadas en el 2018, ya que ingresan nuevos equipos que demandan una curva de aprendizaje mediana y mediana alta, lo que puede explicar los primeros años de la ejecución subnacional, sumado a lo señalado en el párrafo anterior.

Entonces, queda claro que el poder ejecutivo debería fortalecer los sistemas administrativos, simplificarlos y establecer mecanismos de mitigación de riesgos de corrupción, que permitan a la ciudadanía contar con la infraestructura necesaria para una adecuada y efectiva prestación de servicios públicos. Es conveniente resaltar el binomio infraestructura y servicio público. Habrá que preguntarnos si son útiles las infraestructuras si no se cuenta con los recursos para financiar la operación y mantenimiento. $\mathrm{O}$ teniendo el financiamiento, no se cuente con oferta de profesionales calificados para operar y mantener dicha infraestructura, o teniéndolo no se cuente con personal calificado para brindar el servicio, o brindándolo este no cubra los estándares mínimos de calidad.

Entonces, vemos que la infraestructura constituye una condición necesaria para brindar el servicio público, pero no suficiente. El Estado debe — sin duda - procurar generar nuevos mecanismos de ejecución rápida de los proyectos de inversión pública, y es en ese contexto que los contratos gobierno a gobierno - conocidos también como G2G (government to government), por sus siglas en inglés_-, han sido explorados como una opción importante para alcanzar dicho fin, dado el cumplimiento de los cronogramas de ejecución de la infraestructura necesaria para los Juegos Panamericanos y Parapanamericanos 2019, los que se ejecutaron en gran medida, a través de este mecanismo. Pero, cuidado, que la infraestructura creada para los juegos no tiene el nivel de complejidad ni cumple la misma finalidad que, por 
ejemplo, construir hospitales, colegios o una línea de metro.

El gobierno anterior entendía que la solución al déficit de infraestructura económica y social podía ser resuelto a través de este tipo de modalidad de ejecución de proyectos de inversión, aunque sea artificialmente, y a pesar de que el servicio de gestión sea bastante más elevado que el que le cuesta al Estado, lo que no necesariamente es malo, sino que más bien sirve de parámetro para medir el nivel de gasto que realiza el Estado en capital humano y, sobre todo, el nivel de gasto que no realiza para fortalecer al capital humano de la administración pública, asociado a los servicios que se dejan de atender y que a la larga termina siendo más costoso para este, sea porque le resta bienestar a la población (por falta de infraestructura social) o porque el país pierde competitividad (por la ausencia de infraestructura económi$\mathrm{ca})$, que hace que los grandes inversores privados elijan como destino de inversión a nuestros competidores miembros de la Alianza del Pacífico, como Colombia o Chile.

De ahí la importancia de reflexionar sobre si los acuerdos de gobierno a gobierno resultan el mecanismo más idóneo para la ejecución de la infraestructura social y económica, y si en todos los casos se justifica el costo de tener que contratar a otro Estado que supla nuestras deficiencias que, fundamentalmente, son de capacidad de gestión, es decir, de capital humano.

Ahora bien, los acuerdos G2G tienen su origen en el Perú hace ya largos años y no precisamente en el sector infraestructura - como fue el caso de los Juegos Panamericanos 2019- sino más bien en el sector defensa, sobre todo para las compras de equipamiento militar. Se justificaban dichas adquisiciones, de alguna manera, a través de esta modalidad, dada la naturaleza secreta o confidencial en la que suelen realizarse este tipo de adquisiciones en el mundo.

No obstante, de todas las características que tiene un acuerdo G2G, nos enfocaremos en tres que consideramos las más resaltantes:

\section{a) Por el financiamiento del contrato}

Los contratos de gobierno a gobierno exigen contar con el presupuesto corriente, es decir, necesitan tener el dinero en las cuentas del tesoro público, como ocurre con cualquier contratación de adquisiciones de bienes $\mathrm{u}$ obra pública. Y si el contrato superara el ejercicio presupuestal, requerirá el compromiso de la oficina de presupuesto (o la que haga sus veces) de comprometer dichos recursos en los ejercicios subsiguientes. O, caso contrario, haber concertado una operación de endeudamiento que permita financiar los bienes o infraestructura contratados.

Sin embargo, en el caso de los contratos de APP (Asociación Público Privada), como es el caso de las concesiones, una de las principales razones de su dinamismo en Europa en la década de 1990, en el siglo pasado, durante el mandato del primer ministro británico John Major, fue precisamente la necesidad de financiamiento por la que atravesaba el Reino Unido y, al mismo tiempo, la enorme brecha de infraestructura existente; por lo que tuvo que recurrir al financiamiento privado y que el Estado lo pague en el transcurso del tiempo, siendo estas las primeras concesiones de infraestructura de servicios públicos, en la etapa contemporánea.

Entonces, una diferencia trascendental entre ambas formas de ejecución de inver- 
siones, es precisamente las espaldas financieras con las que cuenta el tesoro público. Concretamente hoy, nuestros ahorros son casi nulos, pues el Fondo de Estabilización Fiscal (FEF) ha sido utilizado casi en su totalidad para mitigar los efectos de la crisis económica provocada por la COVID-19.

En ese sentido, en un contexto como el actual, si bien tenemos aún buena capacidad de endeudamiento dados los últimos treinta años de manejo prudente y responsable de las finanzas públicas, es un dato importante a tener en cuenta antes de decidir embarcarse en un contrato de gobierno a gobierno.

No obstante, hace unos días se ha suscrito el acuerdo G2G con el gobierno francés para la ejecución de la carretera central, el mismo que con estudios muy básicos tiene un costo superior a los once mil millones de soles, sin considerar los altísimos riesgos geológicos que involucra dicho proyecto por la geografía en la que se desarrollará. Entonces, dado el nivel de estudios, ese proyecto por lo menos se duplicará, con alta expectativa de elevarse, por metrados, por túneles, por desfase de precios (los estudios son del año 2018).

Dicho esto, en un contexto de caída de ingresos y múltiples necesidades de financiamiento para enfrentar la pandemia, tanto por los costos de adquisición de vacunas como de pruebas de detección, así como por la necesidad de seguir ampliando la oferta sanitaria, no parece ser la mejor decisión asumir un compromiso tan caro, existiendo otras prioridades por el momento.

\section{b) Por los riesgos asociados}

En los contratos de gobierno a gobierno, los riesgos existentes respecto de los bienes o infraestructura a adquirir se restringen únicamente a estos, incluidos los vicios ocultos. Si nos referimos a la construcción de una infraestructura, entonces, el riesgo que asume el contratista es por la construcción de esta, pero luego si el Estado no la mantiene con estándares adecuados, y luego se deteriora, entonces se abrirá un espacio de discusión para determinar quién fue el responsable: si la entidad, porque no realizo el mantenimiento de acuerdo a los estándares de calidad, o la empresa constructora, por vicios de construcción.

En el caso de un contrato de APP, como una concesión, al tratarse de un contrato complejo por tener múltiples prestaciones, los riesgos asociados también son múltiples, y deben ser asignados a la parte que se encuentre en mejor posición para administrarlos eficientemente. Normalmente, el Estado traslada el riesgo de diseńo, de construcción y de financiamiento a la empresa privada. De tal manera que si, durante la operación y mantenimiento de la infraestructura, ocurriera algún problema con la infraestructura, sea causado por un defecto de diseño o de construcción o de ausencia de mantenimiento adecuado, es la empresa privada la responsable de solucionarlo y recuperar los niveles de servicio de acuerdo al contrato.

Entonces, podemos observar que, si una entidad pública decidiera optar por construir la infraestructura, a través de un G2G, y la operación y mantenimiento a través de una APP, entonces, se pierden las bondades de una APP en la etapa previa a la operación del servicio. 
En primer lugar, si bien el riesgo de construcción se mantiene con el constructor de la obra, al entregar dicha infraestructura en concesión, ingresa un sujeto adicional, lo que complica la clarificación del responsable del daño, pues, por un lado, el constructor dirá que fue culpa del concesionario, y este dirá que fue culpa del constructor. Mientras tanto, el Estado se ve privado de brindar el servicio a la población, siendo esta la perjudicada en última instancia.

En segundo lugar, el riesgo de financiamiento es absorbido en su totalidad por el Estado, afectando sus ratios globales de deuda, y el costo de oportunidad de ejecutar otro tipo de proyectos con los recursos que se tienen que reservar para este fin.

En ese sentido, consideramos que no resulta conveniente combinar ambas modalidades de contratación, dado los riesgos que esto implica para el servicio, como por los costos, por ejemplo.

\section{c) Por la calidad del servicio prestado}

Como ya habíamos mencionado, en un contrato G2G no existe un servicio público a cargo del Estado contratante. En efecto, el Estado recibe únicamente la infraestructura y el equipamiento, de corresponder, pero dicha infraestructura la operaría el Estado, en cuyo caso, el nivel de calidad del servicio será el que al Estado le alcance con el financiamiento que se le asigne anualmente, que suele ser un presupuesto modesto; por lo que, rápidamente se verá reflejado en la calidad del servicio, salvo que se decida concesionarla, ya que los niveles de servicio que se exigen al concesionario suelen ser mayores a los que el Estado ofrece a la ciudadanía.

En cambio, en el caso de un contrato de APP, como es el caso de una concesión, el nivel de calidad de servicio es el indicado en las normas técnicas y, ante la ausencia de estas, en lo establecido en el contrato de concesión y en las bases del proceso de promoción de la inversión privada que dio origen al mismo.

En el caso de la nueva carretera central, si fuera concesionada, el Estado está asumiendo todos los riesgos que suelen ser transferidos a la empresa privada, tales como: riesgo de diseño, construcción, financiamiento (en realidad ya no es riesgo sino compromiso firme), operación y mantenimiento. Sin duda, se trata de una obra postergada por décadas, pero es el peor momento y la peor forma de iniciarla en plena pandemia.

Entonces, considerando las diferencias expuestas, y salvo que se trate de una infraestructura que no sirva para brindar un servicio público como el de los Juegos Panamericanos 2019, no resulta conveniente para el Estado realizar contrataciones de gobierno a gobierno para la ejecución de infraestructura pública, pues asume una serie de riesgos que deben ser absorbidos por el Estado (Garcés, 2021)

\section{Conclusiones}

1. El Perú enfrenta una crisis en la ejecución de la inversión pública, agudizada en el último quinquenio, a pesar del ritmo que había obtenido en el año 2015 con récord de ejecución histórica cercana al $90 \%$. 
2. A pesar de las modificaciones del marco normativo del sistema de inversión pública (invierte.pe que reemplazó al SNIP), sin mayores cambios estructurales, ha tenido un ritmo de ejecución muy modesto.

3. Considerar como el principal problema de la ejecución de la inversión pública al sistema de la inversión pública (que motivó su cambio), sin darle un enfoque holístico, constituye un error fundamental, ya que la inversión pública involucra a varios sistemas administrativos, y en cada uno de estos existen cuellos de botella y problemática específica, que, si no son solucionados, mejorados o fortalecidos, así exista la mejor de las normas en materia de inversión pública, esta no despegará.

4. Entre los sistemas administrativos vinculados a la ejecución de la inversión pública, se encuentran: el sistema de planificación, inversión pública, presupuesto, endeudamiento, abastecimiento (contrataciones del estado), tesorería, recursos humanos y control gubernamental.

5. Los sistemas de recursos humanos y de control gubernamental son transversales a todos los sistemas. Siendo fundamental el de recursos humanos para el desarrollo eficiente y eficaz de los sistemas administrativos orientados al cierre de brechas de infraestructura y de provisión de los servicios públicos. En el caso del control gubernamental, es clave para minimizar los riesgos de corrupción durante las distintas fases de ejecución de los proyectos de inversión.

6. Utilizar los contratos de gobierno a gobierno, bajo la premisa de la problemática en la ejecución de la inversión pública, constituye únicamente una justificación para no enfrentar los problemas advertidos, y tiene como efectos mantener la debilidad de capacidades, incrementar los riesgos de diseño, construcción, operación, mantenimiento de las infraestructuras, contribuir al debilitamiento institucional, hipotecar la credibilidad del Perú de un manejo responsable de las finanzas públicas y afectar la sostenibilidad fiscal.

7. La experiencia de los Juegos Panamericanos y Parapanamericanos del 2019 no es comparable con la ejecución de infraestructura para uso de servicio público, dado que el primero tiene fines deportivos con un bajo costo de mantenimiento, mientras que la operación y mantenimiento de una carretera, un colegio o un hospital demandan altos costos de mantenimiento, al mismo tiempo que los riesgos de construcción son mucho más altos.

\section{Bibliografía}

BENITES, J. y Garcés, A. "Inversión Pública en Centroamérica y República Dominicana”. Mimeografía. Washington D. C.: Banco Interamericano de Desarrollo, 2018.

GARCÉS, Alfonso. Contratos Gobierno a Gobierno ¿Constituyen un mecanismo eficiente de ejecución de infraestructura? Revista Gestión Pública \& Control de Gaceta Jurídica. Febrero, 2021.

ORGANISMO SUPERVISOR DE LA CONTRATACIONES DEL ESTADO (OSCE). ¿Por qué razones un proceso de selección queda desierto? Delimitación de causales. Lima: Dirección de Supervisión, Fiscalización y Estudios Subdirección de Estudios Económicos y de Mercado, 2011. https://www.mef.gob.pe/ cont_estado/documentos/1_Estudio_desiertos.pdf 